\title{
Video-Assisted Thoracoscopic Surgery for Treatment of Chronic Obstructive Pulmonary Disease
}

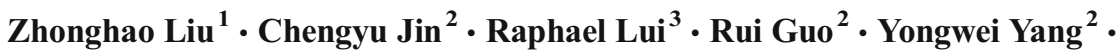 \\ Xiaokaiti Maimaitiyiming ${ }^{2} \cdot$ Chuanliang Peng $^{4}$
}

Received: 7 April 2015 / Accepted: 18 August 2015 / Published online: 28 August 2015

(C) Association of Surgeons of India 2015

\begin{abstract}
The aim of this study is to evaluate the safety and efficacy of lung volume reduction surgery (LVRS) by videoassisted thoracoscopic surgery (VATS) in the treatment of chronic obstructive pulmonary disease (COPD). A total of 90 patients with COPD from 2002 to 2012 were enrolled into our study, comprising 22 who underwent conventional thoracotomies and 68 VATS. Pulmonary function testings, arterial blood gases analysis, and quality of life between these two groups were compared. VATS was found to be superior to the conventional thoracotomy in terms of length of hospital stay, intraoperative blood loss, intubation time, volume of chest tube drainage, and postoperative pain assessment $(P<0.05)$. However, significant differences in pulmonary function testings, arterial blood gases analysis, 6-min walking distance (6-MWD), and postoperative quality of life between the two groups were not found $(P>0.05)$. LVRS by VATS is a safe and reliable surgical approach for the treatment of COPD, with less invasiveness and shorter hospital recovery time.
\end{abstract}

Keywords Video-assisted thoracoscopic surgery $\cdot$ Chronic obstructive pulmonary disease $\cdot$ Lung volume reduction surgery $\cdot$ Thoracotomy

Chuanliang Peng

pechuliang@126.com

1 Department of Orthopedics, the Second Hospital of Shandong University, Jinan, China

2 Department of Thoracic Surgery, Xinjiang Uygur Autonomous Region People's Hospital, Urumqi, China

3 Department of Thoracic Surgery, Cedars-Sinai Medical Center, Los Angeles, CA, USA

4 Department of Thoracic Surgery, the Second Hospital of Shandong University, Jinan, China

\section{Introduction}

Chronic obstructive pulmonary disease (COPD) is a worldwide disease of the respiratory system with significant morbidity and mortality. According to reports from WHO, it will be the third cause of death resulting from smoking in the next 10 years. COPD is characterized by symptoms of progressive dyspnea, hypoxemia, and poor quality of life. If untreated, the 5 -year survival rate is about $25 \%$ [1]. The current medical treatment for COPD is primarily palliation of symptoms, and surgical treatment by lung volume reduction surgery (LVRS) represents another option [2]. Traditionally, LVRS was performed via thoracotomy before the advent of video-assisted thoracoscopic surgery (VATS). It is well documented that thoracotomy is commonly associated with many postoperative complications such as prolonged mechanical ventilation and compromised respiratory efforts due to incisional pain and rib spreading, resulting in atelectasis and pneumonia. Patients with severe COPD are often nutritionally depleted, and thoracotomy incisions in this group of patients are often associated with poor healing. VATS, on the other hand, requires only two or three much smaller incisions and, more importantly, avoids rib spreading, thus offering a safer approach for LVRS. From 2002 to 2012, 90 patients with end-stage COPD underwent LVRS in our department utilizing either the conventional thoracotomy or VATS approach. The clinical outcomes of these two groups of patients were compared.

\section{Methods}

\section{Patients}

Ninety patients who presented to our department with severe COPD between June 2002 and May 2012 were enrolled in the 
study. Based on the patients' preferences and surgeons' experience, 22 and 68 patients subsequently underwent LVRS by thoracotomy and VATS, respectively. The diagnoses of COPD in all the patients were made utilizing commonly employed criteria [3]. The demographics of the patients in both groups showed no significant difference $(P>0.05)$ (Table 1). All the patients had three to four (mean 3.4) episodes per year of exacerbation of COPD due to emphysema-related lung infection, spontaneous pneumothorax, or acute respiratory failure. Inclusion criteria into the study included the following: (1) symptoms of productive cough, shortness of breath, and difficulty breathing; (2) physical examination showed barrel chest and absence of upper lung field breath sounds. Chest X-ray showed wide intercostal space, sparse lung marking, and low and flat diaphragm; (3) chest highresolution computerized tomography (HRCT) and pulmonary ventilation-perfusion scintigraphy (ECT) examination showed "targets" (no lung marking or different size of bulla) (Fig. 1); and (4) dyspnea index classification (MRC revised) of grade III or IV. According to ASA score, five cases were in level II and 17 in level III in thoracotomy group, and 17 in level II and 55 in level III in VATS group. According to Goldman's index of cardiac risk, 15 and 45 cases were above 13 scores in thoracotomy and VATS group, respectively; there were no differences between them $(P<0.05)$. For patients with poor preoperative nutrition, intravenous nutrition support was necessary to ensure normal albumin $(35-47 \mathrm{~g} / \mathrm{L})$ and blood biochemistry. Aspirin should be stop taking the day before surgery (17 cases), and low-molecular-weight heparin would be given subcutaneously after operation. Feeding was the preoperative optimalization for surgery. Preoperative pulmonary function testing, arterial blood gases analysis, and 6-MWT were routinely obtained. Since VATS is a well-established procedure, institutional review board permission was not required. Individual patient consents for surgery (thoracotomy vs VATS) were obtained after the pros and cons of both surgical approaches were explained to the patients.

Table 1 The comparison of general information in two groups

\begin{tabular}{lccc}
\hline Group & Thoracotomy & \multicolumn{1}{l}{ VATS } & $P$ value \\
\hline Age (year) & $63.12 \pm 9.85$ & $66.23 \pm 9.29$ & 0.232 \\
FEV1 (L) & $0.96 \pm 0.17$ & $0.98 \pm 0.11$ & 0.462 \\
$\mathrm{VC} \mathrm{(L)}$ & $1.88 \pm 0.18$ & $1.86 \pm 0.11$ & 0.489 \\
FEV1/VC (\%) & $51.05 \pm 11.51$ & $52.60 \pm 10.05$ & 0.576 \\
$\mathrm{TLC} \mathrm{(L)}$ & $4.17 \pm 0.28$ & $4.28 \pm 0.19$ & 0.059 \\
$\mathrm{PaO}_{2}$ (mmHg) & $60.68 \pm 5.76$ & $62.11 \pm 5.27$ & 0.287 \\
$\mathrm{PaCO}_{2}(\mathrm{mmHg})$ & $47.45 \pm 9.90$ & $48.41 \pm 10.95$ & 0.718 \\
6-MWT (m) & $288.68 \pm 38.08$ & $268.83 \pm 52.54$ & 0.128 \\
\hline
\end{tabular}

\section{Surgical Approach}

Preoperative preparation included the following: (1) Nutritional status, degree of hydration, and electrolyte balance were evaluated and corrected if significant deficiencies were found; (2) reduce the amount of steroid intake if on; and (3)administration of appropriate antibiotics based on sputum cultures if evidence of acute bronchitis exists.

Surgery was performed under general anesthesia. In addition, in some patients, continuous high epidural anesthesia was supplemented so that the amount of inhalation anesthetic drugs could be reduced, thus facilitating recovery from anesthesia and reducing postoperative pain. After a standard prep and drape, a 2-centimeter incision was made in the fourth intercostal space in the anterior axillary line. Dense adhesions were often encountered. Blunt dissection and cautery were used to free up some of the dense adhesions so that the trocar and $30^{\circ}$ thoracoscope could be placed through the eighth space in the midaxillary line. An utility incision was made in the seventh intercostal space in the posterior axillary line or one intercostal space lower for lower resection. In some cases, another $1-\mathrm{cm}$ incision was made in the auscultatory triangle. Stapler was placed through the utility incision to resect the emphysema bullae guided by the preoperative imaging. The range of resection was less than $20-30 \%$. Stapler with a gasket could be used to avoid or minimize air leak if the target area is too large. The incision in the fourth intercostal space could be extended anteriorly for $4 \mathrm{~cm}$ if there were significant intrathoracic adhesions. Ventilator parameters, needed to be adjusted to increase respiratory rate, reduce tidal volume oxygen flow to prevent margin leak. At the end of the procedure, two drainage tubes were placed through two incisions, one to the apex of the thoracic cavity and the other to the costophrenic sinus.

Standard posterolateral thoracotomy incisions were employed in all 22 study subjects. The incision was made between 15 and $20 \mathrm{~cm}$ in length and in the fifth intercostal space. The thoracic cavity was opened layer-by-layer, and the ribs were retracted by a thoracic retractor. Adhesions were taken down if any. Endo-GIA or the conventional ligation approach was used for resection of the bullae. Prior to termination of the procedures, selective intercostal nerve blocks were performed as necessary.

\section{Inspection Indicators}

The intraoperative and postoperative (2 months after operation) observations were prospectively recorded in detail and extensively compared between the two groups, including the length of hospital stay; intraoperative blood loss; intubation time; volume of chest tube drainage and postoperative pain assessment, pulmonary function testing, arterial blood gases analysis, and 6-min walking distance (6-MWD). Pulmonary 
Fig. 1 CT scan showed the "target" of different size of bulla

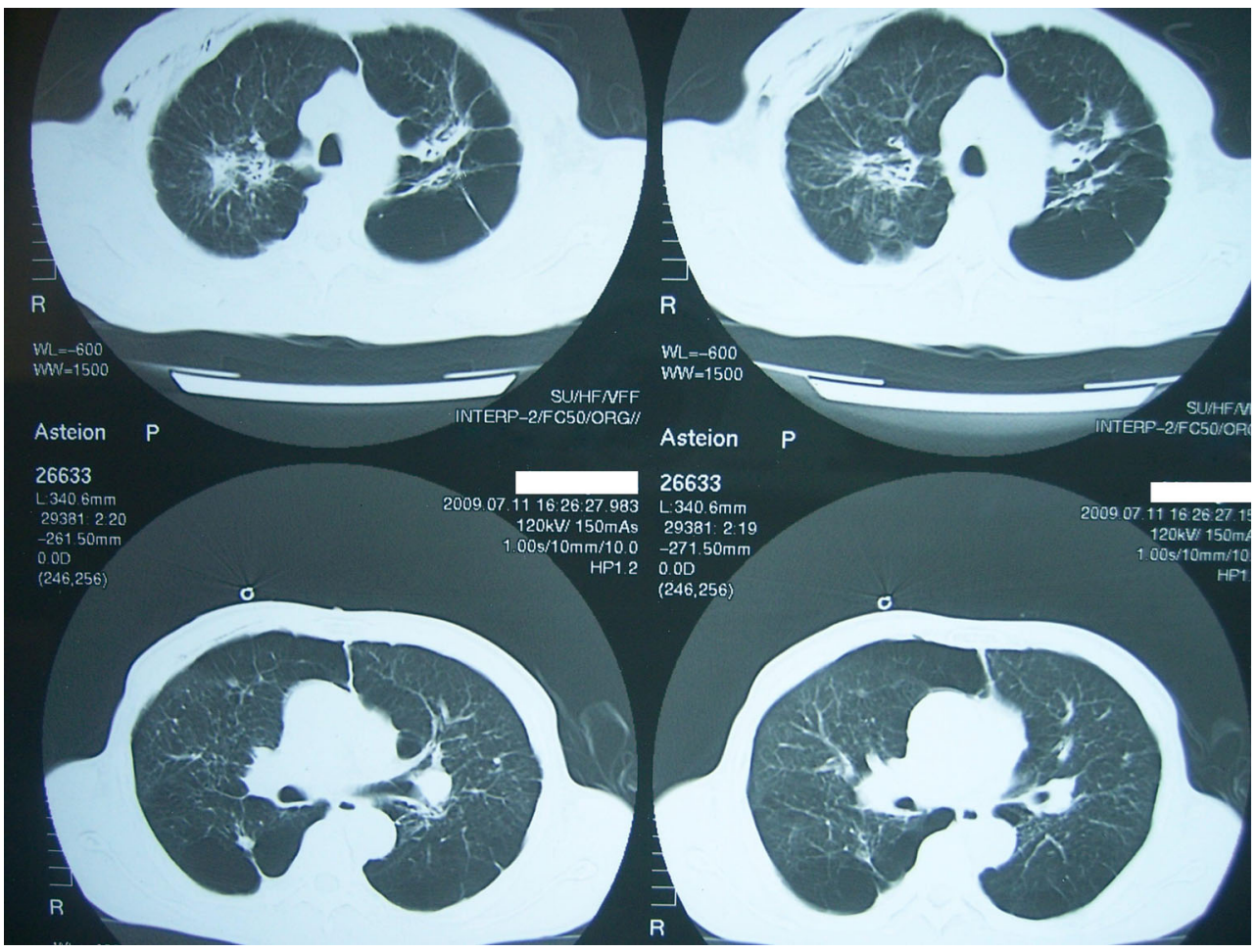

function testing (BF-2 electronic spirometer) includes forced expiratory volume in $1 \mathrm{~s}$ (FEV1), total lung capacity (TLC), and vital capacity (VC).

\section{Quality of Life Assessment}

Life quality was compared between the two groups during the follow-up study. At 6 months postoperatively, quality of life was evaluated with the St. George's respiratory questionnaire (SGRQ) [3]. The survey questionnaire consists of 50 questions, covering three aspects, which involve respiratory symptoms of cough, sputum, chest distress, etc.; activity limitation involving walking up and down and housework and job administration; and social psychological influence of the disease involving anxiety, pain, and disappointment and other negative psychological emotion. All patients in two groups independently completed the surveys, and no suggestion from the outside world was given during the investigation. Score $=$ (sum of positive scores of this part/full score of this part) $\times 100$. Total score $=$ (sum of all positive score/full score of all questions $) \times 100$. The score is inversely correlated with the better of life quality.

\section{Statistical Analysis}

All quantitative data were expressed as mean \pm SD. SPSS 13.0 software (SPSS Inc., Chicago, IL, USA) was applied to analyze single factor analysis of variance and $q$ test. $P<0.05$ was considered statistically significant.

\section{Results}

All patients were sent to the intensive care unit after surgery and mechanically ventilated for 4 to $231 \mathrm{~h}$ (Mean 115) before extubation. All they were discharged in 7 30 days postoperatively. CT scans prior to discharge showed that all the targets had been removed (Fig. 2).

In the VATS group, the incidence of complication was $43.98 \%$ including 23 cases of persistent pulmonary air leak ( $\geq 7$ days, 6 of grade I, 13 of grade II, and 4 of grade III), 13 of arrhythmia, 8 of pulmonary infection, 8 of extensive subcutaneous emphysema, and 3 of acute respiratory failure. Three patients needed mechanical ventilation for more than 7 days. Four patients died of postoperative respiratory failure and infection. Postoperative pulmonary function testing, arterial blood gases analysis, and 6-MWD showed significant improvement compared to their preoperative counterparts $(P<0.05)$. In the thoracotomy group, the incidence of complication was $48.23 \%$ including nine cases of air leak ( $\geq 7$ days, two of grade I, five of grade II, and two of grade III), four of arrhythmia, five of pulmonary infection, four of extensive subcutaneous emphysema, and two of acute respiratory failure. Two patients died of postoperative respiratory failure and infection. Postoperative pulmonary function testing, arterial blood gases analysis, and 6-MWD showed significant improvement compared to their preoperative counterparts $(P<0.05)$. However, there was no significant difference in postoperative inspection indicators and life quality between the two groups $(P>0.05)$ (Tables 2 and 3$)$. 
Fig. 2 Postoperative CT scan showed that the target areas have been removed

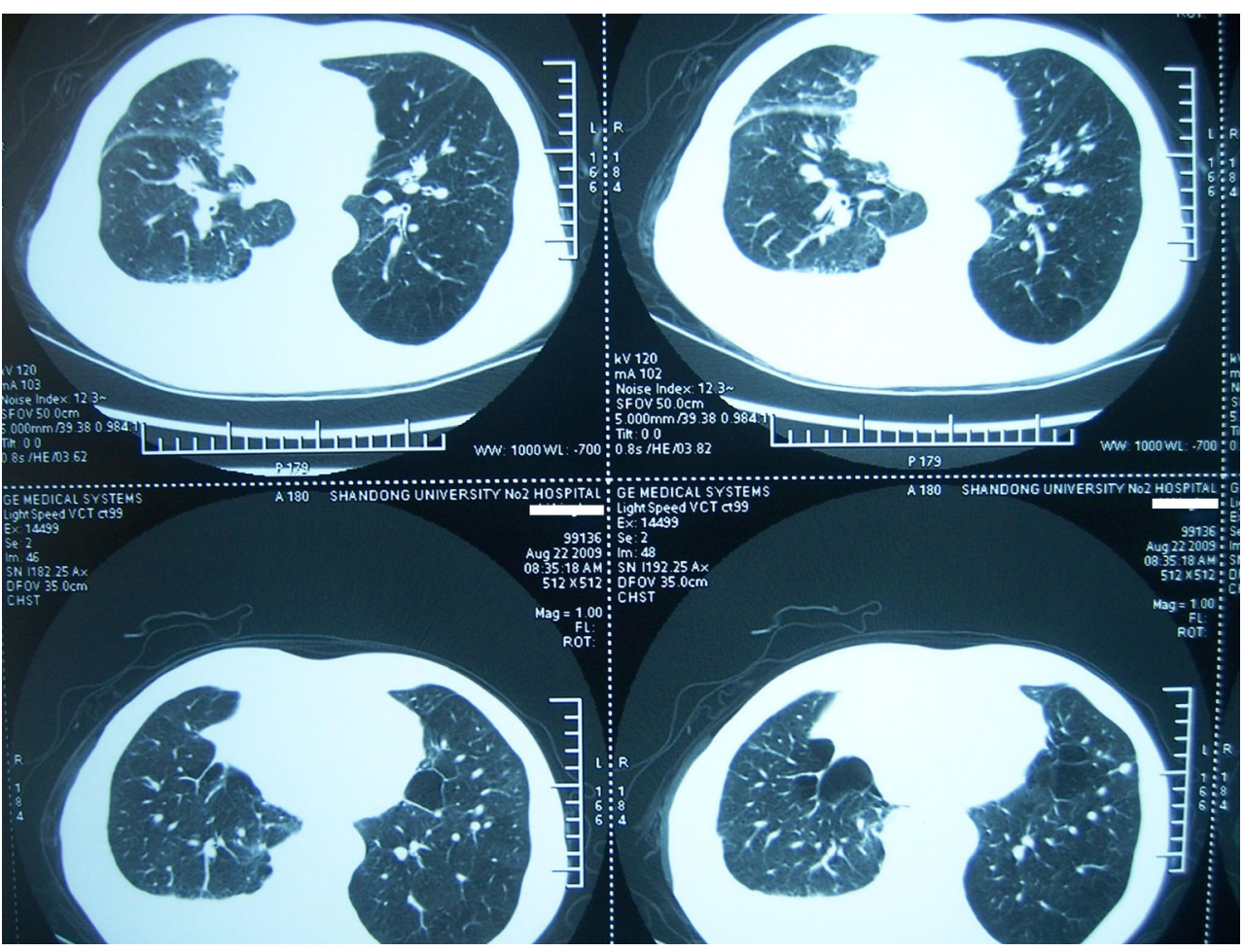

\section{Discussion}

Medical treatment such as antispasmodic and anti-infection for end-stage COPD is not effective for better quality of life. LVRS for COPD is recommended in both China and international guidelines because several trials have shown improvement in survival in selected patients with poor baseline exercise capacity and upper lobe-predominant emphysema [4]. In 1995, Cooper et al. [5] performed LVRS for 20 patients with endstage COPD and achieved demonstrable improved outcomes, so that LVRS as a treatment for COPD was adopted widely in clinical work. Many studies have shown that LVRS can improve pulmonary function, exercise capacity, and quality of life and prolong survival of patients with COPD [6]. To date, there are three different surgical approaches for LVRS: median sternotomy, posterolateral thoracotomy, and VATS. Median sternotomy is often used for bilateral LVRS. Controversy exists over the unilateral or bilateral surgery in one stage or multiple sessions. However, studies indicate that long-term effects of one-side LVRS are similar to that of both sides [7, 8].

VATS, as a minimally invasive surgical approach, was initially used for the treatment of benign diseases and in the
Table 2 The intraoperative and postoperative inspection indicators in two groups

\begin{tabular}{llll}
\hline & Thoracotomy & VATS & $P$ value \\
\hline FEV1 (L) & $1.24 \pm 0.03$ & $1.22 \pm 0.05$ & 0.053 \\
$\mathrm{VC}(\mathrm{L})$ & $2.31 \pm 0.38$ & $2.18 \pm 0.32$ & 0.122 \\
$\mathrm{FEV} 1 / \mathrm{VC}(\%)$ & $53.51 \pm 12.11$ & $55.96 \pm 13.26$ & 0.799 \\
$\mathrm{TLC}(\mathrm{L})$ & $3.81 \pm 0.18$ & $3.87 \pm 0.23$ & 0.227 \\
$\mathrm{PaO}_{2}(\mathrm{mmHg})$ & $89.31 \pm 4.19$ & $88.27 \pm 8.02$ & 0.559 \\
$\mathrm{PaCO}_{2}(\mathrm{mmHg})$ & $33.77 \pm 7.45$ & $32.61 \pm 7.83$ & 0.542 \\
$6-\mathrm{MWT}(\mathrm{m})$ & $415.86 \pm 39.52$ & $422.28 \pm 47.14$ & 0.567 \\
$\mathrm{Hospital} \mathrm{stay} \mathrm{(day)}$ & $16.82 \pm 7.75$ & $13.27 \pm 6.52$ & 0.0455 \\
Intraoperative blood loss (ml) & $300.89 \pm 208$ & $226.36 \pm 110.51$ & 0.0399 \\
Intubation time (day) & $12.11 \pm 1.27$ & $7.27 \pm 3.05$ & $<0.0001$ \\
Volume of drainage (ml) & $853.31 \pm 38.21$ & $671.32 \pm 40.82$ & $<0.0001$ \\
Pain assessment (mild/ $N$ ) & $3 / 20$ & $52 / 64$ & \\
Survival rate & $20 / 22$ & $60 / 64$ & 0.6518 \\
Complication percentage & $48.23 \%$ & $43.98 \%$ & 0.8895 \\
\hline
\end{tabular}


Table 3 Quality of life in two groups at 6 months postoperatively

\begin{tabular}{llllll}
\hline Group & $n$ & Activity score & Respiratory score & Diseases influence score & Total score \\
\hline Thoracotomy & 20 & $46 \pm 6.76$ & $60 \pm 10.69$ & $39 \pm 6.84$ & $43 \pm 6.22$ \\
VATS & 64 & $47 \pm 9.98$ & $58 \pm 9.12$ & $41 \pm 6.92$ & $41 \pm 6.92$ \\
$P$ value & & 0.536 & 0.563 & 0.280 & 0.219 \\
\hline
\end{tabular}

diagnosis of lung tumors. Today, VATS has been gradually integrated into the surgical treatment of COPD. VATS lobectomy is conducted with the aid of sophisticated devices and a camera attached to the thoracoscope, which is inserted into the pleural cavity via small access ports. However, the tactile input, feedback, and skill of the surgeon can be compromised, which may result in a prolonged duration of surgery and high risk of intraoperative blood loss. In our VATS group, the mean operating time was $141.67 \pm 20.12 \mathrm{~min}$, while that for the thoracotomy group was $150.20 \pm 18.06 \mathrm{~min}$. No statistically significant difference was found $(P<0.05)$. However, VATS was superior to thoracotomy in terms of length of hospital stay, intraoperative blood loss, intubation time, volume of postoperative chest tube drainage, and postoperative pain assessment $(P<0.05)$. Thus, VATS appeared to have significant advantage in patients with poor cardiopulmonary function.

Prolonged air leak is common after LVRS and difficult to solve, and air leak cannot be completely avoided even if stapler is used. A large air leak can cause infection, prolong healing, and even result in respiratory failure. Fibrin glue can often be applied on the surface of the lung to achieve a better seal. Mechanical pleurodesis can also be achieved by creating abrasions on the surface of the parietal pleura and promote pleural adhesions. Since varying degrees of air leak were found in almost all the patients after LVRS, appropriately placed chest tubes to the apex of the thoracic cavity and the costophrenic angle are important for adequate drainage of blood and tight apposition of the visceral and parietal pleura. Postoperative nutrition is very important, especially for those who were supported with ventilator. Appropriate antibiotic is necessary based on sputum culture and sensitivity results in case of pulmonary infection.

Our study has confirmed the reported advantages of VATS of less traumatic, faster recovery and shorter hospital stay [4].
However, our survey of the quality of life at 3 months after surgery did not show any significant differences in pulmonary function testing, arterial blood gases analysis, 6-min walking distance (6-MWD), and postoperative quality of life between the two groups.

\section{Funding None.}

Conflict of Interest Author Zhonghao Liu, Author Chengyu Jin, Author Raphael Lui, Author Rui Guo, Author Yongwei Yang, Author Xiaokaiti Maimaitiyiming, and Author Chuanliang Peng declare that they have no conflict of interest.

\section{References}

1. Huber CH, Goeber V, Berdat P, Carrel T, Eckstein F (2007) Benefits of cardiac surgery in octogenarians - a postoperative quality of life assessment. Eur J Cardiothorac Surg 31:1099-1105

2. DeCamp MM Jr, McKenna RJ Jr, Deschamps CC, Krasna MJ (2008) Lung volume reduction surgery: technique, operative mortality, and morbidity. Proc Am Thorac Soc 5:442-446

3. Jones PW, Qurik FH, Baveystock CM (1991) The George's respiratory questionnaire. Respir Med 85:25-31

4. Clark SJ, Zoumot Z, Bamsey O et al (2014) Surgical approaches for lung volume reduction in emphysema. Clin Med 14:122-127

5. Krane M, Voss B, Hiebinger A et al (2011) Twenty years of cardiac surgery in patients aged 80 years and older: risks and benefits. Ann Thorac Surg 91:506-513

6. Zahid I, Sharif S, Routledge T, Scarci M (2011) Is lung volume reduction surgery effective in the treatment of advanced emphysema? Interact Cardiovasc Thorac Surg 12:480-486

7. Wan IY, Toma TP, Geddes DM et al (2006) Bronchoscopic lung volume reduction for end-stage emphysema: report on the first 98 patients. Chest 129:518-526

8. Hopkinson NS (2007) Bronchoscopic lung volume reduction: indications, effects and prospects. Curr Opin Pulm Med 13:125-130 